\title{
Associations between nasal characteristics and sleep polygraphic data in patients suspected Obstructive Sleep Apnea*
}

\author{
Kristin Marie Hoven', Hans-Jørgen Aarstad 1,2, Sverre K Steinsvåg 1,2,3 \\ Department of Otolaryngology, Head and Neck Surgery, Haukeland University Hospital, 5021 Bergen Norway \\ 2 Department of Clinical Medicine, Faculty of Medicine and Dentistry, University of Bergen, Bergen, Norway \\ 3 Department of Otolaryngology, Head and Neck Surgery, Sørlandet Hospital, 4604 Kristiansand, Norway
}

Rhinology Online, Vol 3: 79 - 86, 2020
http://doi.org/10.4193/RHINOL/20.005

*Received for publication:

January 23, 2020

Accepted: May 13, 2020

Published: June 3, 2020

\begin{abstract}
Background: The aim of the present study was to explore the relationships between Obstructive Sleep Apnea (OSA), daytime sleepiness, nasal geometry, nasal airflow, and patient- reported sino-nasal symptoms.

Methodology: Six hundred and fifty-one consecutive patients with suspected sleep related breathing disorder (SRBD) were included in the study. OSA was assessed by the Apnea-Hypopnea Index (AHI) and Oxygen Desaturation Index (ODI). Nasal airflow was measured with Peak Nasal Inspiratory Flow (PNIF). Nasal geometry was measured with Acoustic Rhinometry (AR). Sino-nasal symptoms were graded on Visual Analogue Scales (VAS) as nose-VAS scores. Daytime sleepiness was quantified by the Epworth Sleepiness Scale (EpSS).

Results: There were no general relationship between EpSS scores and nasal airflow, nasal geometry, AHI or ODI scores. When the previously shown nose-VAS associations to EpSS were studied by the AHI score levels, the associations were present primarily among patients with AHI below 15. An exception was the nose-VAS question about "general health" where the association was present for all patients. If the patients were divided by $\mathrm{AHI}=15$ as a cut-off point, an EpSS-unique association to reduced "general health" was found only among the patients with high AHI scores. The Minimal Cross Sectional Area in the posterior part of the nose (MCA2) scored inversely to EpSS among all patients, and was in particular present among patients with AHI below 15.
\end{abstract}

Conclusions: OSA seems largely to be a separate entity from nose function, nasal geometry and nose symptoms.

Key words: obstructive sleep apnea, nasal geometry, sino-nasal symptoms, acoustic rhinometry, peak nasal inspiratory flow

\section{Introduction}

Obstructive Sleep Apnea (OSA) is a widespread sleep disorder. The prevalence may depend on associated pathologies, Body Mass Index (BMI), age and gender ${ }^{(1)}$. OSA is characterized by partial or complete airway collapse during sleep with increased upper airway resistance and risk of hypopnea or apnea as consequences ${ }^{(2)}$. Reported symptoms and signs of OSA include loud snoring, restless sleep, shallow breathing with apnea, and excessive daytime sleepiness. Hrubos-Strøm et al. found in 2011 that $24.3 \%$ of middle-aged Norwegians were at high risk of OSA, and that the prevalence of the disorder in the overall population were $16 \%$ as defined by apnea-hypopnea index (AHI) of 5 or above. The corresponding figure for $\mathrm{AHI}$ of 15 or above was $8 \%$

(3). Numerous studies have shown that if untreated, OSA may have diverse and serious consequences. It is associated with increased risk for cardiovascular morbidity and mortality, stroke, type 2 diabetes, neurological and psychiatric deficits as well as automobile accidents ${ }^{(4,5)}$.

The nose plays an important role in sleep. For most healthy subjects, it is the preferred route of sleep breathing, probably because nasal resistance is considerably lower than oral resistance during sleep ${ }^{(6)}$. Reduced nasal patency may have major 
negative impact on sleep. Serrano et al. described a two-fold risk of disturbed sleep in patients with nasal polyposis compared to controls ${ }^{(7)}$. Allergic rhinitis affects sleep ${ }^{\left({ }^{(8)}\right.}$. It has also been described that patients with chronic rhinosinusitis (CRS), frequently involving nasal obstruction, have reduced sleep quality, increased risk of waking up unrested, and increased daytime sleepiness ${ }^{(9)}$.

As nasal patency seems to be a determining factor for sleep quality, the physiologic, pathophysiologic and therapeutically relationship between the two should be expected to be a subject of major scientific attention. So far, this does not appear to be the case. The existing data on relationships and associations are limited and inconclusive. Thus, we still miss investigations that enable scientifically based decisions about if or how to involve the nose in order to improve sleep and SRBD.

In the present investigation we focused on the relationship between the nose parameters patients' self-reported sino-nasal symptoms, nasal airflow, and nasal geometry and the sleep parameters apnea-hypopnea index (AHI) and oxygen saturation index (ODI).

\section{Materials and Methods}

\section{Study population}

The inclusion criteria in this study was suspicion of SRBD. SRBD includes several chronic disorders characterized by excessive daytime sleepiness and witnessed apneas and / or hypopneas and excessive nighttime snoring ${ }^{(10)}$. Six hundred and fifty one (651) patients referred to the Dept. of Otolaryngology, Head and Neck Surgery, Sørlandet Hospital, Kristiansand, Norway were consecutively recruited after informed consent. There were 458 males and 193 females. The mean \pm SD age was $46.5 \pm 12.0$ years (range: $19-81$ years). Furthermore, $14.8 \%$ had an apnea-hypopnea index (AHI) below 5, $85.2 \%$ above. The patients responded to a questionnaire about co-morbidity with answering alternatives YES or NO. The patients also reported number pack-years of smoking, and level of alcohol consumption. Exclusion criteria were previous surgery in the nose and pharynx, systemic inflammatory disorders like Wegner and sarcoidosis, drug induced rhinitis, current or previous malignancies in the upper airways. Topical nasal steroids and antihistamines were stopped 1 month and 1 week prior to investigation respectively.

\section{Study design}

This is a monocentric cross sectional study.

\section{Respiratory polygraphy - Apnea-hypopnea index (AHI)} All patients underwent a standard respiratory polygraphic recording during sleep using type 3 portable monitors (Embletta or NOX T3, Resmed Norway AS). The following parameters were recorded: plethysmography, nasal flow (nasal cannula pressure transducer), $\mathrm{SpO}_{2}$, respiratory movements (abdomen and

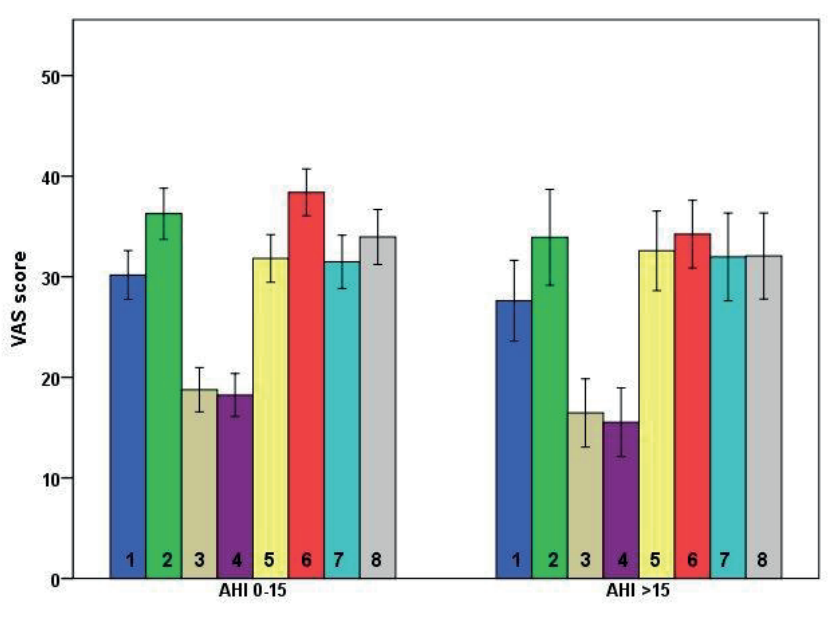

Error Bars: $95 \% \mathrm{Cl}$

Figure 1. NOSE-VAS scores dependent on AHI scores below versus above 15 . Shown are mean $\pm 95 \%$ confidence interval of NOSE-VAS scores with the following order from left to right: 1 . Nasal secretion, 2. headache, 3. pain, 4. sinusitis, 5. coughing, 6 . sneezing, 7. general condition and 8. decreased sense of smell. No significant difference was detected dependent on group allocation.

thorax), snoring, ECG, pulse, and position (11). Scoring rules were recorded accordance to the 2007 American Academy of Sleep Medicine manual, defining apnoea's as a reduction of $90 \%$ or more of baseline nasal airflow with a reduction of at least 10 seconds. Hypopneas were defined as a nasal flow reduction of $30-90 \%$ of baseline, lasting at least 10 seconds accompanied by an oxygen desaturation of $>4 \%$. Diagnosis and severity of OSA was graded based on apnea-hypopnea index (AHI); as no OSA $(<5)$, mild (5-14.9), moderate (15.0-29.9) or severe (>30.0) ${ }^{(12)}$.

\section{Peak nasal inspiratory flow (PNIF)}

PNIF is a portable flow meter to assess nasal resistance and gives a direct measure of nasal obstruction. Youlten Peak Flow meter consists of a face mask which the patients applies over the nose with the mouth closed and measures the peak nasal flow of air during forced inspiration. The results of three maximal inspirations were recorded and the mean of these results are taken as the PNIF ${ }^{(13)}$

\section{Acoustic rhinometry (AR)}

$A R$ is based on reflection of sound waves transmitted into the nasal cavity and are reflected back to create a cross-sectional map of areas of nasal obstruction as a function of the distance from the nostrils. Nasal geometry and volume for various regions in the nose is measured and we can identify the narrowest part of the nasal cavity or minimal cross-section area (MCA) ${ }^{(14,15)}$. An impulse acoustic rhinometer [RhinoMetrics SRE2100 (Rhinoscan version 2.5, built 3.2.5.0)] was handled by 3 trained operators. Procedures were in accordance with published proto- 

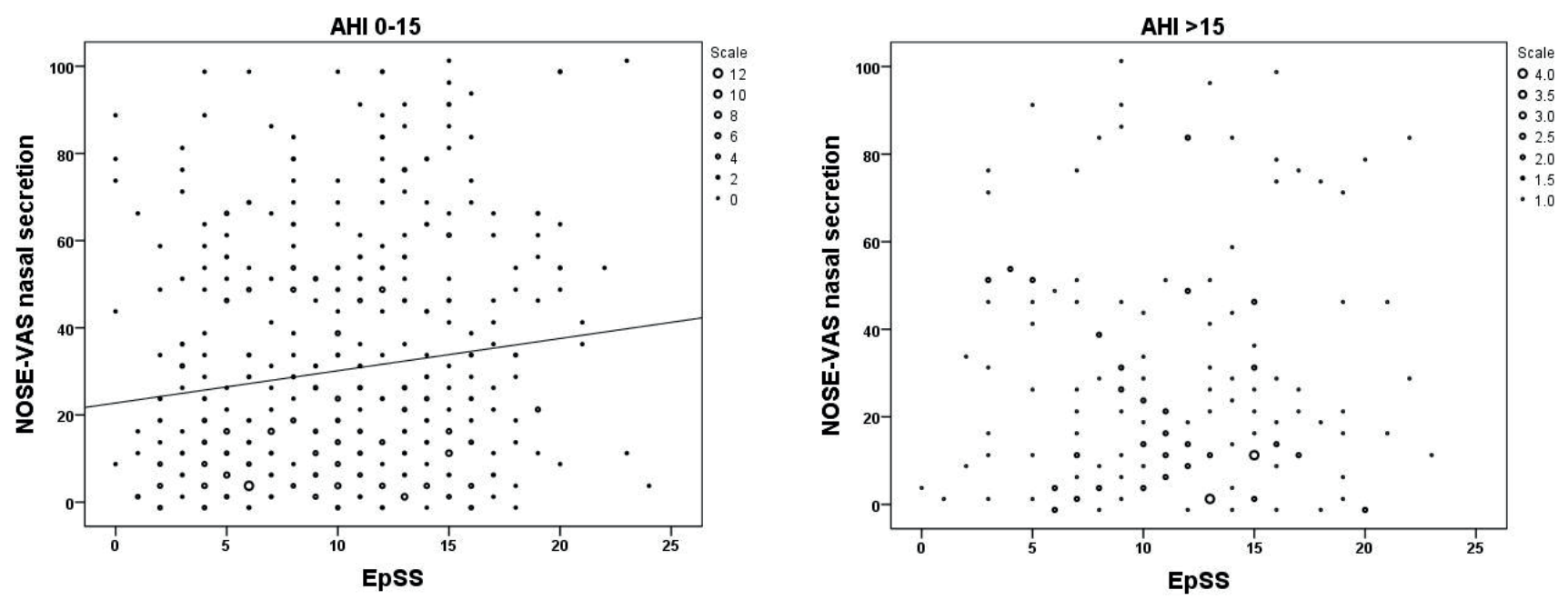

Figure 2. Scatter plots showing NOSE-VAS nasal secretion scores by EpSS scores dependent on AHI scores below versus above 15. Statistics (Pearson Correlation): (AHI 0-15): $r 2=0.019 ; p<0.01$. A regression line has also been constructed according to the formula: $Y=23+0.74 * x .(A H I>15)$ : not significant.

cols, with the mean of 3 consecutive measurements of volumes and minimal cross sectional areas in each nasal cavity, before and after decongestion with oxymetazoline ${ }^{(16)}$.

\section{Nose symptoms on Visual analogue scales (Nose-VAS)}

Nose-VAS was used to quantify the subjective feeling of nasal obstruction and other sino-nasal related symptoms. Patients were asked to put a mark on a $100 \mathrm{~mm}$. linear scale ranging from no obstruction/no symptoms to complete obstruction/mostintense symptoms possible. In the present study we include the following 9 symptoms; Nasal obstruction, nasal secretion, headache, mid-facial pain, coughing, sneezing, sense of smell, complains with sinusitis as well as general health ${ }^{(17,18)}$.

\section{Epworth sleepiness scale psychometry (EpSS)}

EpSS is a simple and rapid method for measuring degree of daytime sleepiness. EpSS is a patient reported measure of sleepiness ${ }^{(19)}$. It presents eight different situations about sleepiness that are commonly met in daily life. A higher total score (range $0-24)$ indicates increased degree of sleepiness ${ }^{(20)}$. The employed EpSS cut-off score separating those with normal versus excessive level of daytime sleepiness is $11^{(21)}$.

\section{Statistics}

We used a commercially available statistical program package (IBM Corp. Released 2012. IBM SPSS Statistics for Windows, Version 24.0 Armonk, NY, USA). Figures are reported as mean \pm standard err of the mean (SEM) or mean \pm standard deviation (SD). Statistical significance was considered if $p<0.05$. All $\mathrm{P}$-values reported represent two-sided tests. Cronbach alpha, Pearson correlation, Partial correlation, Factor analysis and Student t-test were calculated as indicated.

\section{Results}

\section{Patient characteristics}

Of the 651 included patients 191 (29.3\%) stated to have allergy, 59 (9.1 \%) asthma, 133 (20.4\%) hypertension, 49 (7.5 \%) cardiac disease and 22 (3.4\%) kidney disease. In addition, 339 (52.1 \%) reported to be on any medication.

\section{Associations between Nose-VAS scores, AR and PNIF and AHI levels}

Using ANOVA analyses, the nose-VAS scores depended on $\mathrm{AHI}$ scores, both divided by AHI levels (results not shown) and divided by $\mathrm{AHI}$ lower or higher than 15 (Figure 1). No significant difference between the groups was observed.

\section{Correlation between Nose-VAS scores and ESS/AHI index/ ODI scores}

As previously published, a significant correlation was shown between the following VAS-nose scores and the EpSS score: "Nasal secretion", "headache", "coughing," "sneezing" and "general condition". Studying the nose-VAS scores, only level of "headache" correlated significantly but inversely with the AHI index ( $r=$ $-0.08 ; p<0.05)$. Regarding the ODI scores, none of the VAS-nose question responses correlated significantly (Table 1).

If separating the patients by EpSS score levels, the different nose-VAS scores lost significant correlations from present among low AHI scoring patients to absent among high $\mathrm{AHI}$ scoring patients by four steps of AHI levels defining no, mild, moderate to serious OSA. This was particularly evident comparing patients with no versus serious OSA (Table 2). Regarding the "general condition" the response levels were significantly correlated in the $\mathrm{AHI}$ 0-5 group and the $\mathrm{AHI}$ above 30 group (Table 2). We also studied the correlations between the nose-VAS scores 
Table 1. Pearson correlation coefficients between Epworth sleeping scale (EpSS), Apnoe Hypanoe Index (AHI), Oxygen desaturation Index (ODI), gender, age, rhinometry, and NOSE-VAS scores.

\begin{tabular}{|c|c|c|c|c|c|c|}
\hline & EpSS & AHI & ODI & Gender & Age & BMI \\
\hline $\mathrm{AHI}$ & $.14^{* * *}$ & & & & & \\
\hline ODI & $.19^{* * *}$ & $.90^{* * *}$ & & & & \\
\hline Gender ( $0=$ female). & -.05 & $.09^{*}$ & $.09^{*}$ & & & \\
\hline Age & -.01 & $.18^{* * *}$ & $.18^{* * *}$ & -.06 & & \\
\hline BMI & $.14^{* *}$ & $.30^{* * *}$ & $.37^{* * *}$ & -.06 & .05 & \\
\hline Nasal congestion & .03 & -.04 & -.01 & .05 & $-.17^{* * *}$ & .03 \\
\hline Nasal secretion & $.10^{*}$ & -.04 & -.03 & .02 & .04 & -.04 \\
\hline Headache & $.12^{* *}$ & $-.08^{*}$ & .02 & $-.18^{* * *}$ & $-.09^{*}$ & $.08^{*}$ \\
\hline Mid-facial pain & .04 & -.06 & -.04 & $-.08^{*}$ & -.03 & .06 \\
\hline Sinusitis & .02 & -.08 & .05 & -.06 & -.06 & .06 \\
\hline Coughing & $.09^{*}$ & -.01 & .02 & -.02 & .00 & .06 \\
\hline Sneezing & $.09^{*}$ & -.08 & -.03 & .00 & .00 & .05 \\
\hline Decr. sense of smell & .07 & -.04 & -.00 & $.11^{* *}$ & $-.13^{* *}$ & -.04 \\
\hline Decr. gen. condition & $.15^{* * *}$ & .01 & .04 & -0.6 & .05 & $.11^{* *}$ \\
\hline MCA1 & .02 & -.02 & -.00 & $.08^{*}$ & .01 & .03 \\
\hline MCA2 & $-.10^{*}$ & .02 & -.02 & -.02 & -.01 & -.00 \\
\hline PNIF & -.05 & -.06 & -.05 & .01 & -.03 & .05 \\
\hline
\end{tabular}

${ }^{*}=\mathrm{P}<0.05,{ }^{* *}=\mathrm{P}<0.01,{ }^{* * *}=\mathrm{P}<0.001 ; \mathrm{MCA}$ : nasal cavity Minimal Cross-section Area; PNIF: Peak nasal inspiratory flow.

and the EpSS scores in patients with AHI below and above 15 (Table 3). Then, correlations between the nose-VAS scores and the EpSS scores were as in the analyses when all patients were included in the group with AHI scores below 15. On the other hand, in the group of patients with $\mathrm{AHI}$ above 15 there were no such relationships (Table 3)(Figure 2). One exception was noted regarding "general condition" which scored significantly at the same level in both $\mathrm{AHI}$ groups.

A significant correlation was determined between EpSS and the $\mathrm{AHI}$ index $(r=0.14 ; \mathrm{p}<0.001)$ when including all patients (Table 1). When studying divided groups based on $\mathrm{AHI}$ scores with a cutoff point of 15 , only among patients with a high $\mathrm{AHI}$ score, a significant correlation was shown (Table 3).

In the above-mentioned analyses, the associations between levels of "nasal congestion" and the other nose-VAS scores were systematically included as positive control analyses in order to show that the nose-VAS interaction scores stayed the same among all patients. This was indeed the case.

Correlation between EpSS and nose-VAS scores dependent on age, gender and present comorbidity

Present comorbidity, in particular asthma, but also allergy, cardiac disease and hypertonia were associated with increased score on the nose-VAS questions (Table 4). The same was the case with positive answers to "whether on any medication". Partial correlation analyses were subsequently performed between the EpSS and nose-VAS scores adjusting in step 1 by age and gender of the patient and in step 2 by any additionally reported comorbidities (Table 5). It was shown that including information about co-morbidities explained some, but not all-common variance between the EpSS and the nose-VAS scores. This was also valid when splitting the patients in groups with $\mathrm{AHI}$ scores below or above 15 (Table 5).

In addition, the "nasal congestion" scores were used as positive controls in order to exclude changed internal association between the nose-VAS scores dependent on AHI score levels. The controls performed as expected.

Correlation between acoustic rhinomanometry (AR), peak nasal inspiratory flow (PNIF) and nose-VAS measures Results from AR and PNIF were correlated with EpSS, AHI and ODI scores. Including all patients, MCA2 correlated to EpSS scores $(r=-0.10 ; p<0.05)$ (Table 1). If studied by AHI level score groups, this association was found only among patients scoring AHI below15 (Table 3).

\section{Discussion}

In the present paper, we have studied the association between self-reported nasal obstruction and self-reported nasal complaints given on VAS, nasal airflow measured by PNIF, nasal geometry measured by AR and the sleep parameters $\mathrm{AHI}$ and ODI in 651 patients with the suspicion of SRBD. There were no 
Table 2. Pearson correlations between NOSE-VAS scores and Epworth sleepiness scores (EpSS) with «nasal congestion» scores as "positive" control; divided by AHI score level groups.

\begin{tabular}{|c|c|c|c|c|c|c|c|c|}
\hline \multirow[b]{2}{*}{ NOSE-VAS questions about: } & \multicolumn{2}{|c|}{ AHI 0-5 } & \multicolumn{2}{|c|}{ AHI 6-15 } & \multicolumn{2}{|c|}{ AHI 16-30 } & \multicolumn{2}{|c|}{ AHI 31 - } \\
\hline & Epss & $\begin{array}{c}\text { Nasal } \\
\text { congestion }\end{array}$ & Epss & $\begin{array}{c}\text { Nasal } \\
\text { congestion }\end{array}$ & Epss & $\begin{array}{c}\text { Nasal } \\
\text { congestion }\end{array}$ & Epss & $\begin{array}{c}\text { Nasal } \\
\text { congestion }\end{array}$ \\
\hline Nasal congestion & .06 & & -.06 & & .09 & & .03 & \\
\hline Nasal secretion & $.22^{* * *}$ & $.25^{* * *}$ & .02 & $.26^{* *}$ & .13 & .07 & -.05 & $.31^{* * *}$ \\
\hline Headache & $.13^{*}$ & .02 & $.30^{* *}$ & $.24^{* *}$ & -.00 & .08 & .09 & $.18^{*}$ \\
\hline Mid-facial pain & .05 & .12 & .12 & $.24^{* *}$ & .06 & .13 & -.07 & -.05 \\
\hline Sinusitis & .08 & $.22^{* * *}$ & .04 & $.24^{* *}$ & .03 & $.26^{* *}$ & -.13 & .13 \\
\hline Coughing & .05 & $.30^{* * *}$ & .13 & .14 & $.20^{*}$ & .17 & .01 & $.35^{* * *}$ \\
\hline Sneezing & $.13^{*}$ & $.21^{* * *}$ & -.02 & .06 & $.22^{*}$ & $.24^{* *}$ & .01 & $.20^{*}$ \\
\hline Decreased sense of smell & $.12^{*}$ & $.37^{* * *}$ & .00 & $.21^{*}$ & .12 & $.35^{* * *}$ & .02 & $.41^{* * *}$ \\
\hline Decreased general condition & .12 & $.16^{* *}$ & .17 & .14 & .18 & .11 & .16 & $.23^{*}$ \\
\hline No. respondents in group & 260 & 267 & 122 & 131 & 113 & 128 & 117 & 123 \\
\hline
\end{tabular}

${ }^{*}=\mathrm{P}<0.05,{ }^{* *}=\mathrm{P}<0.01,{ }^{* * *}=\mathrm{P}<0.001 ; \mathrm{MCA}$ : nasal cavity Minimal Cross-section Area; PNIF: Peak nasal inspiratory flow; "Nasal congestion" scores as positive control.

Table 3. Pearson correlation coefficients between Epworth sleepiness scale (EpSS)/AHI/"nasal congestion" score and VAS-Nose scores with groups by low versus high AHI score.

\begin{tabular}{|c|c|c|c|c|c|c|c|c|}
\hline & \multicolumn{4}{|c|}{ AHI 0-5 } & \multicolumn{4}{|c|}{ AHI 16- } \\
\hline & Epss & AHI & ODI & $\begin{array}{c}\text { Nasal } \\
\text { congestion }\end{array}$ & Epss & AHI & ODI & $\begin{array}{c}\text { Nasal } \\
\text { congestion }\end{array}$ \\
\hline $\mathrm{AHI}$ & -.03 & & $.675^{* * *}$ & & $.22^{* *}$ & & $.806^{* * *}$ & \\
\hline ODI & $.12^{*}$ & & & & & & & \\
\hline Nasal congestion & .04 & $-.13^{* *}$ & -.08 & & .03 & -.03 & .04 & \\
\hline Nasal secretion & $.14^{* *}$ & -.00 & .02 & $.21^{* * *}$ & -.00 & -.02 & -.03 & $.30 * * *$ \\
\hline Headache & $.15^{* *}$ & -.02 & .02 & .08 & .08 & -.13 & -.02 & $.19^{*}$ \\
\hline Mid-facial pain & .08 & -.07 & .00 & $.15^{* *}$ & -.04 & -.05 & -.01 & .03 \\
\hline Sinusitis & .06 & -.04 & -.02 & $.24^{* * *}$ & -.07 & -.14 & -.06 & .15 \\
\hline Coughing & $.10^{*}$ & -.04 & .02 & $.25^{* * *}$ & .04 & -.05 & .02 & $.28^{* * *}$ \\
\hline Sneezing & $.12^{*}$ & $-.10^{*}$ & -.10 & $.20 * * *$ & .02 & -.03 & -.08 & .15 \\
\hline Decreased sense of smell & $.10^{*}$ & -.05 & .01 & $.35^{* * *}$ & .01 & -.02 & .05 & $.31^{* * *}$ \\
\hline Decr. gen. condition & $.15^{* *}$ & .03 & .08 & $.16^{* * *}$ & .15 & -.00 & .03 & .15 \\
\hline MCA1 & -.05 & -.07 & -.02 & $-.10^{*}$ & .11 & .00 & .00 & -.01 \\
\hline MCA2 & $-.10^{*}$ & .05 & $-.11^{*}$ & $-.15^{* *}$ & -.09 & .06 & -.04 & -.12 \\
\hline PNIF & -.07 & -.03 & -.06 & $-.12^{*}$ & -.00 & -.00 & -.06 & $-.27^{* *}$ \\
\hline
\end{tabular}

${ }^{*}=\mathrm{P}<0.05,{ }^{* *}=\mathrm{P}<0.01,{ }^{* * *}=\mathrm{P}<0.001$. "Nasal congestion" scores as positive control.

general significant associations between the nasal parameters on one the hand and AHI/ODI levels on the other.

Previous investigations on this are not conclusive. Some investigators have employed the sino-nasal-outcome-test (SNOT-20) to explore the issue and claimed that patients suffering from OSA have a reduced sino-nasal-quality of life compared to controls ${ }^{(22,23)}$. SNOT-20 is a validated, self-administered quality of life instrument specific for symptoms of rhinosinusitis and sensitive to clinical changes. It describes the health burden of rhinosinusitis by measuring physical problems, functional limitations and emotional consequences of CRS by asking the participants to score 20 core symptoms. Few of the above mentioned SNOT versus OSA studies have employed ideal control groups, i.e. control and experimental groups closely related to each other. We 
Table 4. Pearson correlations between Nose-VAS scores and reported comorbidity.

\begin{tabular}{|c|c|c|c|c|c|}
\hline Nose-VAS questions about: & Allergy & Asthma & Cardiac disease & Hypertonia & Any medication \\
\hline Asthma & $.30^{* * *}$ & & & & \\
\hline Cardiac disease & .02 & $.15^{* * *}$ & & & \\
\hline Hypertonia & .06 & $.11^{* *}$ & $.18^{* * *}$ & & \\
\hline Any medication & $.18^{* * *}$ & $.19^{* * *}$ & $.15^{* * *}$ & $.35^{* * *}$ & \\
\hline Nasal congestion & $.13^{* * *}$ & $.13^{* *}$ & -.01 & -.01 & .015 \\
\hline Nasal secretion & $.12^{* *}$ & $.13^{* *}$ & $.13^{* *}$ & .07 & $.08^{*}$ \\
\hline Headache & .07 & $.12^{* *}$ & .03 & .08 & $.13^{* *}$ \\
\hline Mid-facial pain & $.16^{* * *}$ & $.19^{* * *}$ & $.11^{* *}$ & $.09^{*}$ & $.12^{* *}$ \\
\hline Sinusitis & $.18^{* * *}$ & $.16^{* * *}$ & $.09^{*}$ & .03 & $.12^{* *}$ \\
\hline Coughing & .04 & $.17^{* * *}$ & $.09^{*}$ & .05 & $.10^{*}$ \\
\hline Sneezing & .05 & $.09^{*}$ & $.09^{*}$ & .03 & .02 \\
\hline Decreased sense of smell & .07 & $.08^{*}$ & .05 & .05 & -.01 \\
\hline Decreased general condition & .07 & $.13^{* *}$ & $.09^{*}$ & $.11^{* *}$ & $.21^{* * *}$ \\
\hline
\end{tabular}

Table 5. Partial correlations scores between None-VAS scores and Epworth sleepiness scores (EpSS) including indicated control variables.

\begin{tabular}{|c|c|c|c|c|c|c|c|c|}
\hline \multirow[b]{3}{*}{ Nose-VAS questions about: } & \multicolumn{4}{|c|}{ Age, gender } & \multicolumn{4}{|c|}{$\begin{array}{c}\text { Age, gender, allergy, asthma, cardiac disease \& } \\
\text { hypertonia \& kidney disease, any medication, pack- } \\
\text { years smoking, BMI }\end{array}$} \\
\hline & \multicolumn{2}{|c|}{ AHI 0 - 15} & \multicolumn{2}{|c|}{ AHI 15- } & \multicolumn{2}{|c|}{ AHI 0 - 15} & \multicolumn{2}{|c|}{ AHI 15- } \\
\hline & EpSS & $\begin{array}{c}\text { Nasal } \\
\text { congestion }\end{array}$ & EpSS & $\begin{array}{c}\text { Nasal } \\
\text { congestion }\end{array}$ & EpsS & $\begin{array}{c}\text { Nasal } \\
\text { congestion }\end{array}$ & EpsS & $\begin{array}{c}\text { Nasal } \\
\text { congestion }\end{array}$ \\
\hline Nasal congestion & .01 & & .08 & & .01 & & .08 & \\
\hline Nasal secretion & $.15^{* *}$ & $.27^{* * *}$ & .04 & $.21^{* *}$ & $.15^{* *}$ & $.27^{* * *}$ & .04 & $.21^{* *}$ \\
\hline Headache & $.22^{* * *}$ & $.11^{*}$ & .06 & .09 & $.22^{* * *}$ & $.11^{*}$ & .06 & .09 \\
\hline Mid-facial pain & .09 & $.18^{* * * *}$ & .00 & .02 & .09 & $.18^{* * *}$ & .00 & .02 \\
\hline Sinusitis & .08 & $.24^{* * *}$ & -.07 & $.16^{*}$ & .08 & $.24^{* * *}$ & -.07 & $.16^{*}$ \\
\hline Coughing & .09 & $.26^{* * *}$ & .11 & $.23^{* * *}$ & .09 & $.26^{* * *}$ & .11 & $.23^{* * *}$ \\
\hline Sneezing & .09 & $.18^{* * *}$ & .12 & $.26^{* * *}$ & .09 & $.18^{* * *}$ & .12 & $.26^{* * *}$ \\
\hline Decreased sense of smell & .09 & $.31^{* * * *}$ & .06 & $.37^{* * *}$ & .09 & $.31^{* * *}$ & .06 & $.37^{* * *}$ \\
\hline Decreased general condition & $.15^{* *}$ & $.17^{* *}$ & $.19^{* *}$ & $.19^{* *}$ & $.15^{* *}$ & $.17^{* *}$ & $.19^{* *}$ & $.19^{* *}$ \\
\hline
\end{tabular}

«Nasal congestion» scores as "positive" control.

therefore hypothesize that previously published differences may be secondary to the choices of non-parallel groups. As being an important clinical matter, this question needs more scrutiny. We have presently studied our cohort by ANOVA analyses studying the nose-VAS score results dependent on AHI scores, both divided by AHI levels and divided by AHI lower or higher than 15 . No significant differences between the groups were observed. The same was the case studying AR and PNIF results. This argues strongly against an association between $\mathrm{AHI}$ levels on one side and nose-VAS, nasal volumes or nasal function on the other. A hypothesized general association between AHI/ODI and nose-VAS/AR scores implies that such associations should be demonstrated at individual levels, i.e. with significant correlations between these parameters. Presently, this is not the case. We have, however, previously demonstrated a significant correlation between EpSS scores and nasal symptom levels ${ }^{(24)}$. In the present investigation, we have extended this to study if these associations were present to the same extent in the subgroups with and without OSA respectively. We found that the association between EpSS score and the NOSE-VAS questions primarily was present among the patients with $\mathrm{AHI}$ scores below 15 , i.e. patients with not more than mild OSA. These results were independent of age, gender, allergy, asthma, cardiac disease \& hypertonia \& kidney disease, any medication, pack-years smoking, and BMI. The results also gave support to state that these associations decrease stepwise as the $\mathrm{AHI}$ score increases. One notable exception was the question about "general condition". It is possible that this association is coincidental as reduced 
"general condition" may in part also be caused by increased daytime sleepiness. This should be investigated in future studies. The level of "headache" correlated significantly, but inversely with the AHI index, i.e. patients with OSA suffered less from headache than those without. This is in contrast to previous investigations. The term "sleep apnea headache $(\mathrm{SAH})$ " refers to a condition characterized by morning headache with age, male gender and $\mathrm{BMI}$ as predisposing factors ${ }^{(25,26)}$. Oxygen desaturation cannot explain the patho $\neg$ physiology of SAH alone, and CPAP treatment does not seem to improve this headache ${ }^{(27,28)}$. This, combined with our results, questions the overall legitimacy of the term.

There are studies concluding with nasal obstruction as an independent risk factor for OSA. Virkkula et al. found that nasal resistance in non-obese individuals was related to sleep parameters ${ }^{(29)}$. A link between nasal geometry and OSA has also been described by others.

MCA 1 and MCA 2 were significantly smaller in OSA patients compared to controls, and the severity of the sleep disorder was inversely correlated to the nasal dimensions. Inconsistently, we found a weak, and probably coincidental, association between mild OSA $(\mathrm{AHI}<15)$ and MCA 2, i.e. a reduced minimal cross sectional area between 3 and $5,2 \mathrm{~cm}$. from the nasal orifice, compared to individuals with more serious OSA. This corresponds with the dominating opinions about nasal airway morphology and OSA:There are no associations.

With negative results, as in the present study, and results inconsistent with previous investigations, it is important to consider the validity of the studied parameters. We have included more than six hundred patients giving statistical power even to negative conclusions. The matter of skewed questionnaire response patterns from healthy to OSA patients was addressed by studying comparison to other questions. We have systematically used the question about "nasal congestion" as a positive control. As can be seen from the results, a generally skewed response pattern dependent on $\mathrm{AHI}$ can probably be ruled out. We have also shown expected association between present asthma, allergy and cardiac disease and level of nasal symptoms.

Regarding the $\mathrm{AHI}$, we have shown a close correlation between the $\mathrm{AHI}$ and ODI scores as expected. Thus, these two physiologically different measurements, if presently wrongly measured, must be affected similarly. In addition, we have determined an association between $\mathrm{AHI} / \mathrm{ODI}$ on the one side and BMI, gender and age of the patient on the other side as expected. Still, a better quality than presently used may always be achievable as to include parameters.

The present investigation cannot once and for all completely rule out all questions about associations between nasal function and OSA. Several findings still argue in favor of this association. From a nose-anatomical point of view, nasal obstruction during sleep may contribute to SRBD because nasal breathing is more effective than oral breathing, especially during sleep. Supine position may increase nasal resistance through postural reflex mechanisms, and changes in hydrostatic pressure in venous sinusoids ${ }^{(30)}$. Nasal obstruction has also been related to sleep apnea and frequent arousals through upper airway collapse due to more negative oropharyngeal pressure during inspiration ${ }^{(31)}$. From a neuromuscular point of view, there are speculations about decreased sensitivity on nasal mechanoreceptors, particularly prevalent around the nasal vestibule, in OSA-patients ${ }^{(32)}$. They are essential in reflex mechanisms against negative pressure in the upper airways through modulation of the genioglossus muscle activity. Their dysfunctionality may represent a link between the nose and SRBD. From a respiratory point of view, a patent airway depends on a balance between dilating and collapsing forces. The nasal cavity as such is a non-collapsible tube as described in the Starling resistor model. However, due to the mucosal lining, particularly the part covering the inferior turbinates, it is an active tube responsive to temperature, humidity, pollution, emotions, posture, etc. Thus, the narrowest point in the nose may change in position and narrowness. Consequently, there will be changes in airflow and stimulation of mechanoreceptors. This may eventually modulate the collapsible part of the airway, the pharynx, and induce SRDB. Furthermore, nasal disorders like polyps, septal deviations etc. causes mouth breathing. When this happens, the jaw and tongue move backwards and downwards predisposing for airway collapse. Accordingly, there are several mechanisms linking the nose to sleep. However, a most important matter that remains to be understood and explained is why nasal characteristics do not appear to be associated with the most serious form of SRBD, namely OSA. That this really is the case has been confirmed in a number of interventional studies looking at the effect of medical and surgical measures to open the nose on OSA. There is not sufficient data to recommend drug treatment of OSA. Nasal surgery does not appear to be able to reduce OSA severity. Neither does nasal dilators ${ }^{(32)}$.

\section{Conclusions}

Based on more than 650 patients, we conclude that there is no general association between nose patency and AHI or ODI scores. Thus, the claim about a causal relationship between the nose function and OSA has been further weakened. Medical and surgical measures against nasal obstruction are not likely to improve the sleep disorder. Nevertheless, treating nose disease may improve daytime sleepiness, as well as improve adherence to CPAP in its own right.

\section{Acknowledgments}

We thank Irene Winger, Liv Henny Kyllingstad and Anne Cathrine Lønnerød for collecting the study data. 


\section{Authorship contribution}

SS designed the data collection for the study. $\mathrm{KMH}, \mathrm{HJAa}$, and

SS analyzed the data, wrote the manuscript, edited the manuscript and approved the final version.

\section{Conflict of interest}

The authors declare that they have no conflict of interest.

\section{Ethics approval and consent to participate}

Not applicable.

\section{Consent for publication}

Not applicable.

\section{Availability of data and materials}

Not applicable.

\section{Funding}

Not applicable.

\section{References}

1. Senaratna CV, et al., Prevalence of obstruc tive sleep apnea in the general population: A systematic review. Sleep Med Rev, 2017 34: 70-81

2. Destors M, et al., [Pathophysiology of obstructive sleep apnea syndrome and its cardiometabolic consequences]. Presse Med, 2017.

3. Hrubos-Strom H, et al., A Norwegian population-based study on the risk and prevalence of obstructive sleep apnea. The Akershus Sleep Apnea Project (ASAP). J Sleep Res, 2011. 20(1 Pt 2): 162-70.

4. Zhang W, Si LY, Obstructive sleep apnea syndrome (OSAS) and hypertension: pathogenic mechanisms and possible therapeutic approaches. Ups J Med Sci, 2012. 117(4): 370-82

5. Arita A, et al., Risk factors for automobile accidents caused by falling asleep while driving in obstructive sleep apnea syndrome. Sleep Breath, 2015. 19(4): 1229-34

6. Fitzpatrick MF, et al., Partitioning of inhaled ventilation between the nasal and oral routes during sleep in normal subjects. J Appl Physiol (1985), 2003. 94(3): 883-90.

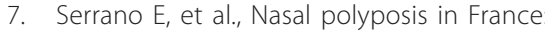
impact on sleep and quality of life. J Laryngol Otol, 2005. 119(7): 543-9.

8. Sundbom, F., et al., Asthma symptoms and nasal congestion as independent risk factors for insomnia in a general population: results from the GA(2)LEN survey. Allergy, 2013. 68(2): 213-9.

9. Bengtsson C, et al., Chronic Rhinosinusitis Impairs Sleep Quality: Results of the GA2LEN Study. Sleep, 2017. 40(1)

10. Burman D, Sleep Disorders: Sleep-Related Breathing Disorders. FP Essent, 2017. 460: 11-21.

11. Nerfeldt P, Aoki F, Friberg D. Polygraphy vs. polysomnography: missing osas in symptomatic snorers--a reminder for clinicians. Sleep Breath, 2014. 18(2): 297-303.

12. Bjorvatn $B$, et al., Prevalence of excessive sleepiness is higher whereas insomnia is lower with greater severity of obstructive sleep apnea. Sleep Breath, 2015. 19(4):
1387-93.

13. Ottaviano G, Fokkens WJ. Measurements of nasal airflow and patency: a critical review with emphasis on the use of peak nasa inspiratory flow in daily practice. Allergy. 2016. 162-74

14. Clement PA, et al., Consensus report on acoustic rhinometry and rhinomanometry. Rhinology, 2005. 43(3): 169-79.

15. de Oliveira Camargo Gomes A, et al. Nasal cavity geometry of healthy adults assessed using acoustic rhinometry. Braz Otorhinolaryngol, 2008. 74(5): 746-754.

16. Hilberg O, Pedersen OF. Acoustic rhinometry: recommendations for technical specifications and standard operating procedures. Rhinol Suppl, 2000. 16: p. 3-17.

17. Kjaergaard T, Cvancarova M, Steinsvag SK, Cigarette smoking and self-assessed upper airway health. Eur Arch Otorhinolaryngol, 2011. 268(2): 219-26.

18. Ottaviano G, et al., Peak nasal inspiratory flow measurement and visual analogue scale in a large adult population. Clin Otolaryngol, 2019. 44(4): 541-548.

19. Hardinge FM, Pitson DJ, Stradling JR. Use of the Epworth Sleepiness Scale to demonstrate response to treatment with nasa continuous positive airways pressure in patients with obstructive sleep apnoea. Respir Med, 1995. 89(9): 617-20.

20. Johns MW, Reliability and factor analysis of the Epworth Sleepiness Scale. Sleep, 1992 15(4): 376-81.

21. Pallesen $\mathrm{S}$, et al., Prevalence and risk factors of subjective sleepiness in the general adult population. Sleep, 2007. 30(5): 619-24.

22. Moxness MHS, et al., Sinonasa Characteristics in Patients with Obstructive Sleep Apnea Compared to Healthy Controls. Int J Otolaryngol, 2017. 2017: 1935284

23. Awad MI, Kacker A, Nasal Obstruction Considerations in Sleep Apnea. Otolaryngol Clin North Am, 2018. 51(5): 1003-1009.

24. Hoven KM, et al., Correlation between Excessive Daytime Sleepiness (EDS) and self-reported and objective nasal characteristics. Rhinology, 2018. 56(4): 316-322.
25. Suzuki K, et al., Sleep apnoea headache in obstructive sleep apnoea syndrome patients presenting with morning headache: comparison of the ICHD-2 and ICHD3 beta criteria. J Headache Pain, 2015. 16: 56

26. Kristiansen HA, et al., Sleep apnoea headache in the general population. Cephalalgia, 2012. 32(6): 451-8.

27. Mitsikostas DD, Vikelis M, Viskos A Refractory chronic headache associated with obstructive sleep apnoea syndrome. Cephalalgia, 2008. 28(2): 139-43.

28. Russell MB, Kristiansen HA, Kvaerner KJ Headache in sleep apnea syndrome: epidemiology and pathophysiology. Cephalalgia, 2014. 34(10): 752-5.

29. Virkkula P, et al., Nasal obstruction and sleep-disordered breathing: the effect of supine body position on nasal measurements in snorers. Acta Otolaryngol, 2003. 123(5): 648-54.

30. Kase Y, Hilberg O, Pedersen OF. Posture and nasal patency: evaluation by acoustic rhinometry. Acta Otolaryngol, 1994. 114(1): 70-4.

31. Suratt PM, Turner BL, Wilhoit SC. Effect of intranasal obstruction on breathing during sleep. Chest, 1986. 90(3): p. 324-9.

32. Shintaro C, Park CS. Establishing a Patent Nasal Passage in Obstructive Sleep Apnea. Sleep Med Clin, 2019. 14(1): p. 41-50.

Kristin Marie Hoven

Department of Otolaryngology

Head and Neck Surgery

Haukeland University Hospital

5021 Bergen

Norway

Tel: +4795067214

E-mail:

kristin.marie.hoven@helse-bergen.no

ISSN: 2589-5613 / @2020 The Author(s). This work is licensed under a Creative Commons Attribution 4.0 International License. The images or other third party material in this article are included in the article's Creative Commons license, unless indicated otherwise in the credit line; if the material is not included under the Creative Commons license, users will need to obtain permission from the license holder to reproduce the material. To view a copy of this license, visit http://creativecommons.org/ licenses/by/4.0/ 